\title{
Pranayama Yoga: Measuring Brainwaves via EEG
}

\author{
Rebecca Bhik-Ghanie \\ Bard College at Simon's Rock, Great Barrington, Massachusetts, rbhikghanie@simons-rock.edu
}

\begin{abstract}
Differences in baseline electroencephalogram (EEG) activity have been found among longtime practitioners of meditation $(3+$ years $)$ in comparison to novice meditators $(<1$ year). In the current study, 10 participants (5 experienced meditators; 5 novices) engaged in a series of weekly, 20-minute Open Heart Meditation (OHM) practices; 5 control participants (all novices) engaged in a 20 -minute reading/study session. EEG activity was measured in all participants during the first and final sessions. While the differences did not reach statistical significance, there were differences in the expected direction, suggesting an increase in baseline mean frequency of EEG measurements within the beta range for experienced meditators in comparison to novices and controls, suggesting an increase in neurological engagement during meditation. These findings suggest that there are potential neurological gains of certain meditative practices, which should lead to further investigation into the merits of meditative practice as a form of therapy.
\end{abstract}

KEYWORDS: EEG, electroencephalogram, brain, yoga, mindfulness, alpha, beta, meditation, OHM

\section{Introduction}

As meditative practices have become more prominent within mainstream health-related practices in the United States, research on the neurological effects of meditative practices has also increased in frequency and findings. Using electroencephalography, magnetic resonance imaging (MRI) and functional magnetic resonance imaging (fMRI), scientists have been able to, often in real-time, measure neurological activity during meditation sessions over prolonged periods of time (e.g., Gard et al., 2014; Grant, Courtemanche, Duerden, Duncan, \& Rainville 2010; Jadhav, Manthalkar, \& Joshi 2017). Recent research has also suggested that individuals may benefit from neurological changes after initiating a meditation practice; benefits include increases in cortical thickness within advanced meditators and greater caudate connectivity (Gard et al. 2015; Lazar et al. 2005). This paper continues this research by further investigating the neurological effects of a series of meditation sessions via electroencephalogram (EEG).

\section{Methods}

\section{Participants and Study Design}

Inclusion criteria for sampling were: (a) being a novice practitioner of meditation (have meditated less than 5 times, or have never successfully completed a 10 minute meditation sequence), or (b) being an experienced practitioner (those who have two or more years of on-or-off meditation, retreat attendees welcomed) (c) committing to at least 3 weeks of Pranayama yoga sessions. An email to the campus community recruited those who were interested in participating.

This criteria was used because prior studies have shown that differences in brainwave activity are more apparent between novice and experienced meditator groups (Gard et al. 2015; Lutz, Greischar, Rawlings, Ricard, \& Davidson 2004; Lutz et al. 2009). The final sample included 10 novice practitioners (randomly assigned to meditation or control group) and 5 experienced practitioners of meditation (all assigned to meditation group).

\section{Procedures $^{1}$}

To obtain baseline measures of brain activity and heart-rate, EEG and pulse tests were administered to all participants during the first and third meditation or control sessions. Participants in the meditation conditions participated in a weekly, 20-minute one-on-one meditation session (with the researcher, a certified yoga-instructor). The three sessions were spaced throughout the week with

1 The study protocol was reviewed and approved by the Bard College at Simon's Rock Institutional Review Board. 
respect to the participants' convenience, and sessions were developed using techniques outlined in Deckersbach, Hölzel, Eisner, Lazar \& Nierenberg's (2014) text, Mindfulness-based cognitive therapy for bipolar disorder.

Themes for meditation sequences were created by the researcher and centered around resilience-building and practicing empathy. In the first session, entitled Intense Feelings in Stressful Situations, participants were asked to visualize a time that brought immense amounts of stress to them, so much that they felt it in their body. They were then asked to exaggerate this feeling and think of it in so much detail that they were almost recreating that moment. After this, they were asked to sit with those feelings for a couple of minutes, and then visualize feelings of warmth targeting the areas of stress in their body. Breath was a tool that was used to "exhale negative feelings". This exercise was intended to teach participants how to manage feelings in intense situations.

In the second session, entitled Extending Kindness, participants were asked to think of someone who has been important in their lives, and to visualize the details of their appearance, mannerisms, and personality. After getting a precise image of this person in their mind, they were asked to think of a time where they made this person overwhelmed with happiness, laughter, or tears. They were asked to focus on this moment, and to then think about the things they have done in their lives to help that person. This exercise was intended to remind participants about empathetic feelings and to appreciate themselves for having the ability to help others.

The third session, entitled Self-care; Personal Assessment, engaged participants in an activity that involved their taking time to evaluate their current state of mind, and how they have been feeling overall for the week. They were then asked to reflect on stressful things in their lives (e.g. work-related or personal) and to welcome those things while maintaining a relaxed state of mindfulness. Participants were asked to go into the details of their bodily processes and to "thank" different organs for supporting them when they may not be tending to their bodies as much as they need to (i.e. during stressful times). Participants were also asked to tell themselves that they deserve a meditative practice, as a treat to themselves. This exercise was intended to expand upon self-love, self-appreciation, and care for functioning bodily processes. Participants in the control group did not engage in meditation sessions and were asked to simply work on homework, study, or read. These participants were given all the same assessments as the meditation group (EEG and pulse).

\section{Measurements and Assessments}

Alpha and beta waves were measured via electroencephalogram (EEG) - the brand of the device was iWorx Systems Inc., model 214. The software used for analysis was LabScribe v2. Five electrode stickers were placed on the skull regions just above specified locations in the frontal, parietal and temporal regions of participants ( 2 on the left and right parietal and temporal regions, and 1 on the frontal regions). Participants wore the 5 electrodes for a total of 2 sessions (the 1 st and 3rd), each running for a duration of 20 minutes each (for an overall total of 40 minutes of EEG readings). Using the EEG on participants is non-invasive and the electrode stickers enable the attachment of the sensor electrodes.

The heart rate of all participants was measured via radial or carotid pulse. Within the meditation and control groups, pulse was assessed at the start and end of first and third sessions to analyze changes in heart rate from the start to the end of each session and across the three-week program.

The EEG data were analyzed using SPSS and the VassarStats.net online website. To compare the effects of meditation/yoga on experienced/novice participants, there were three betweensubjects conditions: (1) Novice Meditators - Exposed to Meditation Sessions; Group, Experienced Mediators - Exposed to Meditation Sessions; , and (3) Control Group (all novices and who were not exposed to any meditation/yoga during the study). All three groups of participants' brain wave frequencies were measured on two occasions: during the first session of yoga/meditation (or control) and during the third session of yoga/meditation (or control). Thus, the Session 1-toSession 3 change in EEG measurements variable was a within-subjects variable. All brainwave 
frequencies were compared across experimental conditions and across sessions using MANOVA analyses. Thus, means were obtained for the frequencies of brain waves-in the alpha and beta waves-two times for each participant: sessions 1 and 3.

\section{Results}

\section{Left Beta Waves}

As shown in Figure 1 below, the mean frequencies of beta waves in the left region of the brain were highest for experienced meditators in the experimental (meditation/control) condition, next highest for novices in the experimental condition, and lowest for those in the control condition. These betweensubject means differed significantly, $\mathrm{F}(2,12)=13.67, \mathrm{p}=.001$; the experienced meditators exposed to meditation in the study had the highest left hemisphere mean beta wave frequency; the novice meditators exposed to meditation had the next highest (and not significantly different from that of the experienced meditators); the control participants had the lowest mean left hemisphere beta wave frequency.

Also, the interaction of condition by session was statistically significant, $F(2,12)=5.18, p=$ .02. What appears to be happening is that both novice and experienced experimental groups increased in left beta wave activity, while the control group did not change.

Figure 1. Left Hemisphere Mean Frequency Beta Waves

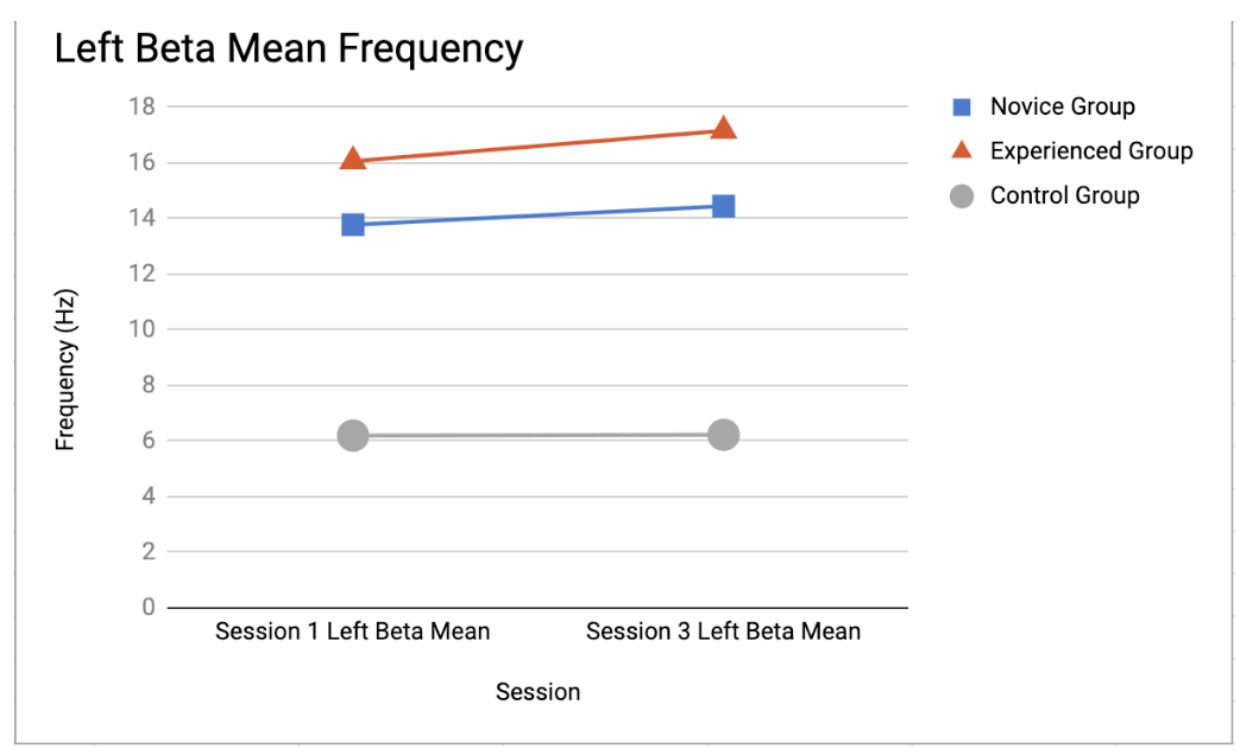

Right Beta Waves

As shown in Figure 2, on the next page, a very different pattern of mean frequencies emerged in the measurements of of beta waves in the right hemisphere of the brain, and the overall differences were not differ statistically, $\mathrm{F}<1$. The interaction of session by condition (novice, experienced/meditation, novice/meditation) were, however statistically significant, $F(2,12)=4.14$, $p$ $<.05$. Interpretation of this interaction effect suggests that the novice group increased in right hemisphere beta wave activity, the experienced group increased moderately, and the control group decreased slightly. 
Figure 2. Right Hemisphere Mean Frequency Beta Waves

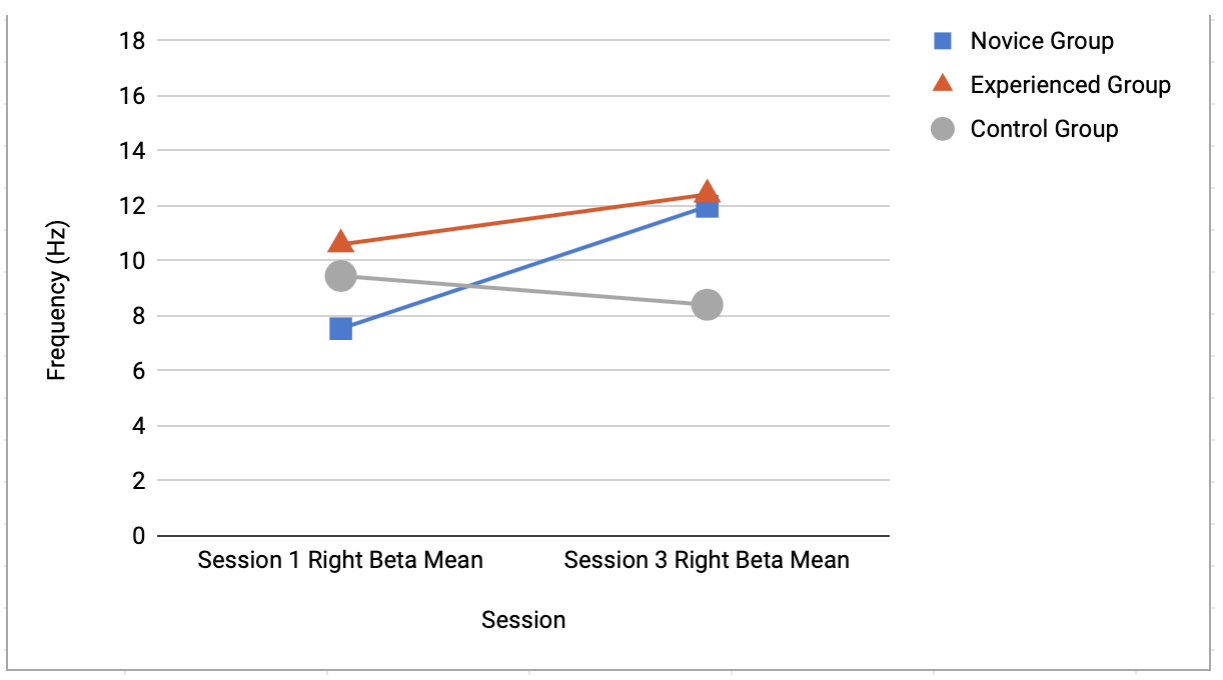

\section{Left Alpha Waves}

As shown in Figure 3 below, the mean frequencies of alpha waves in the left region of the brain were highest for experienced meditators in the experimental (meditation/control) condition, with both the novice and control groups displaying lower mean left hemisphere alpha wave activity. These differences were statistically significant, $F(2,12)=6.23, p<.02$. Also, the interaction of condition by session was marginally significant, $\mathrm{F}(2,12)=3.25, \mathrm{p}<.10$. Review of the means suggests that the experienced experimental group increased in left alpha wave activity, while the novice and control group did not change.

Figure 3. Left Hemisphere Mean Frequency Alpha Waves

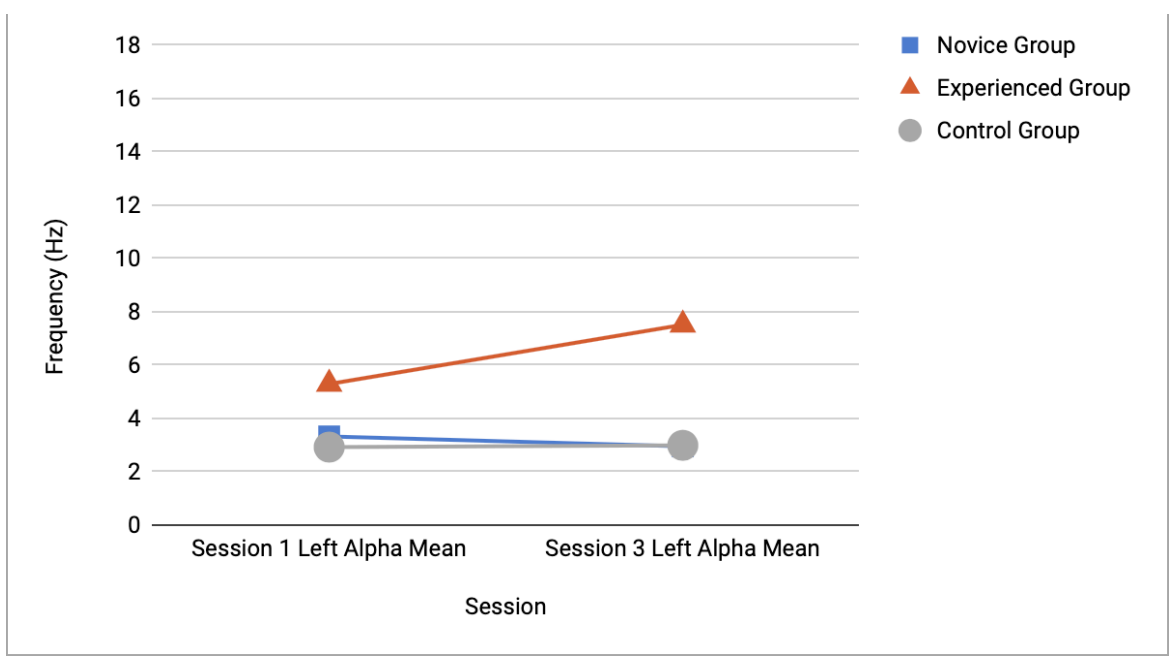

Right Alpha Waves

As shown in Figure 4 below, the mean frequencies of alpha waves in the right region of the brain were highest for experienced meditators in the experimental (meditation/control) condition, next highest for novices in the experimental condition, and lowest for those in the control condition. However, these means did not differ statistically, $\mathrm{F}(2,12)=1.38, \mathrm{p}=.29$. Comparisons of means during session 1 and session 3, and the interaction of session by condition were also not significant, $\mathrm{F}<1$. Although the graph may suggest an interaction effect, the variability within the groups clearly overrode any statistical reliability of the differences. 
Figure 4. Right Hemisphere Mean Frequency Alpha Waves

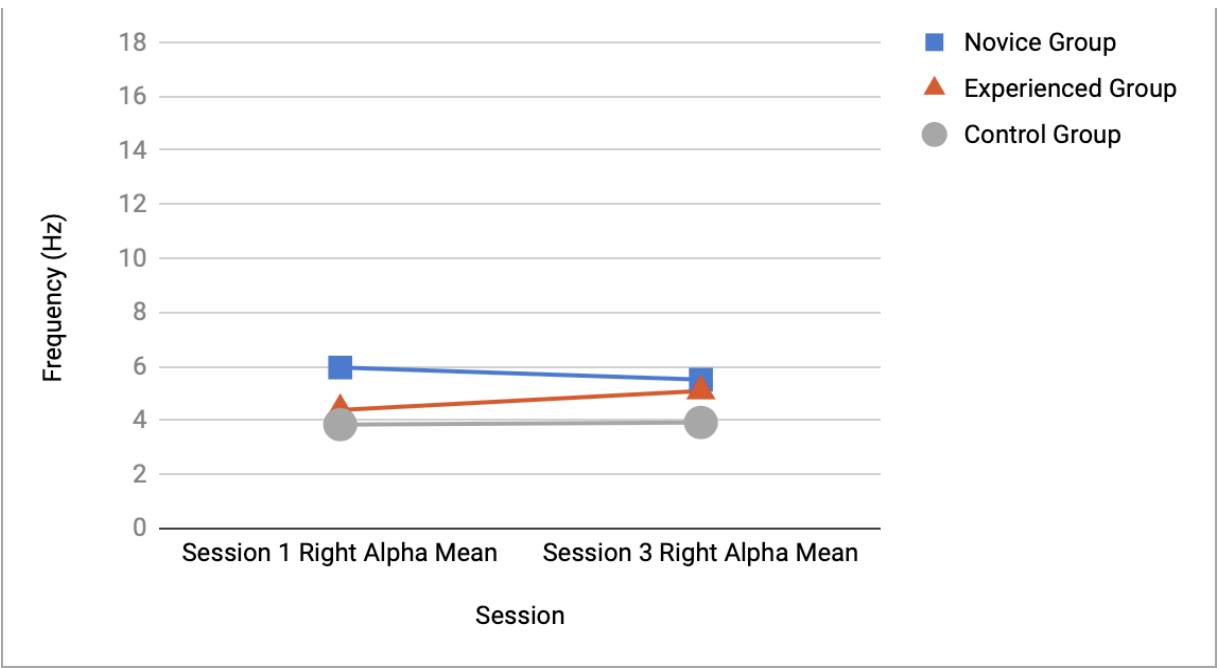

\section{Pulse}

Changes in participants' heart rate are shown in Figure 5 below. This bar graph shows a difference between the average post- and pre-session pulse values for experienced, novice, and control groups for sessions 1 and 3. The mean change in pre-post pulse rates for sessions 1 and 3 within the experienced group are $-3.5 \mathrm{bpm}$ and $-11 \mathrm{bpm}$, respectively (meaning that these participants' pulse rates decreased, on average $3.5 \mathrm{bpm}$ in their first session, and $11 \mathrm{bpm}$ in their third session). The mean post-pre pulse values for session 1 and 3 within the novice group are $-3.8 \mathrm{bpm}$ and $-5 \mathrm{bpm}$, respectively. The mean post-pre values for session 1 and 3 within the control group are increases of $1.4 \mathrm{bpm}$ and $2.6 \mathrm{bpm}$, respectively. This suggests that within the control group, participants' heart rate increased between sessions 1 and 3, and for the experimental groups--those exposed to yoga/meditation-their heart rate decreased between the start and end of each sessions, and this decrease generally was greater after three sessions than on the first session.

Figure 5. Differences in post-pre Pulse measure

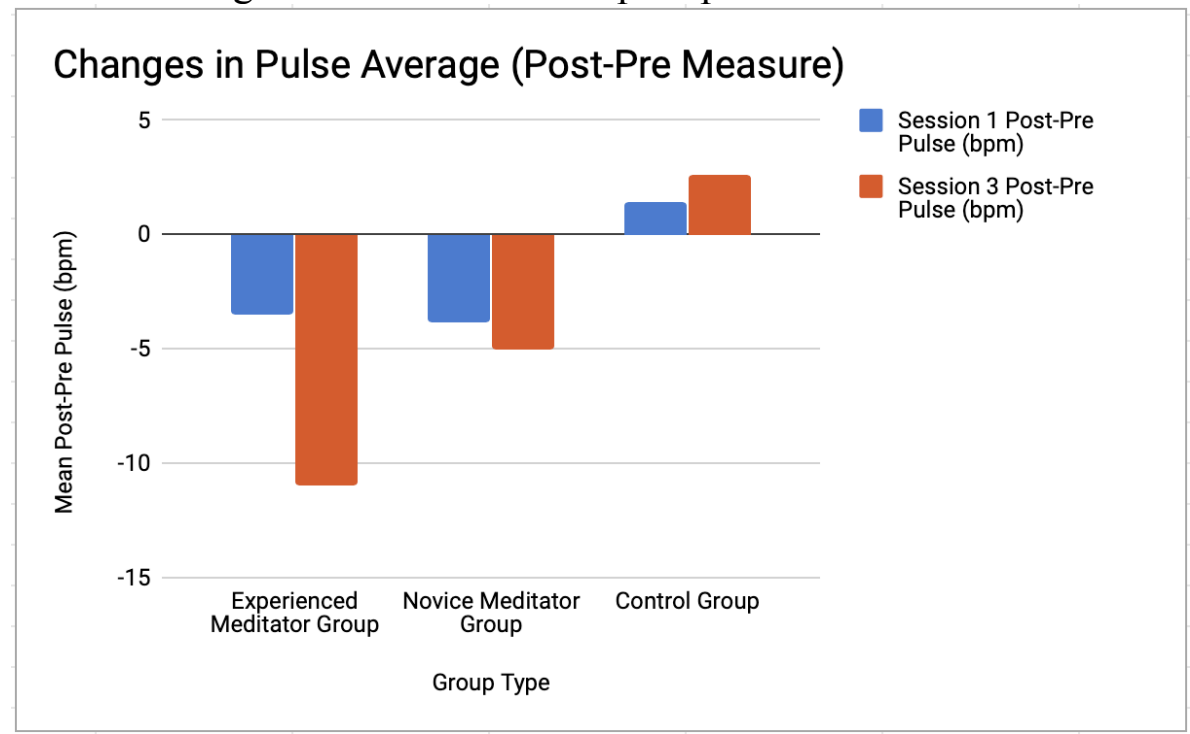

\section{Discussion and Conclusion}

The results are consistent with the predictions on which this study was based. Focusing on the EEG data, novice meditators who were exposed to a three-week meditation session reported, on average, 
an increase in beta wave activity, slightly in the left hemisphere, and especially in the right hemisphere. There was no significant or visible change, on average, in alpha wave activity for in either hemisphere for these participants. The experienced meditators, over the three weeks meditation exercise, showed slight increases in beta wave activity in both hemispheres, but also increases in alpha wave activity in the right hemisphere (no change in alpha wave activity in the left hemisphere). As expected, the control group participants (who did reading/homework, not meditation) basically demonstrated no change in EEG activity, on average, between the three sessions.

Despite the small sample size in this study, these data are, we hope, valuable in that they provide support to other studies which have found that experienced meditators show differences in brain activity in comparison to those in novice meditator groups (Tang, Hölzel, \& Posner 2015; Lee et al. 2015). As noted above, the change in mean frequencies of brain activity in the alpha wave ranges appeared also to support the predictions, but only for the experienced meditators, and only in the right hemisphere.

In the future, we hope to expand this small-sample study to a larger, more robust, sample of participants. Future research might also explore the benefits that pranayama yoga may have for those who face neurological difficulties, as well as physiological challenges, and those who have difficulties maintaining psychological well-being (e.g. anxiety, depression, lack of mental compartmentalization, low resilience). Previous studies show beneficial effects of yoga \& meditation practices in those suffering from these disorders (Deckersbach et al. 2014; Gard et al. 2015; Jadhav et al. 2017; Lee et al. 2015), but there is still much to learn about how these benefits occur and for whom. In addition to these findings, prolonged meditation practitioners have displayed alterations in the physiology of their brain (Gard et al. 2014; Gard et al. 2015; Grant et al. 2010). Research in this area has the potential to reveal many more discoveries about the effects of meditation on the mind.

\section{References}

Deckersbach, T., Hölzel, B., Eisner, L., Lazar, S. W., \& Nierenberg, A. A. 2014. Mindfulness-based cognitive therapy for bipolar disorder. New York: Guilford Press.

Gard, T., Taquet, M., Dixit, R., Hölzel, B. K., de Montjoye, Y.-A., Brach, N., .. Lazar, S. W. 2014. "Fluid intelligence and brain functional organization in aging yoga and meditation practitioners." Frontiers in Aging Neuroscience, 6. https://doi.org/10.3389/fnagi.2014.00076.

Gard, T., Taquet, M., Dixit, R., Hölzel, B. K., Dickerson, B. C., \& Lazar, S. W. 2015. "Greater widespread functional connectivity of the caudate in older adults who practice kripalu yoga and vipassana meditation than in controls." Frontiers in Human Neuroscience, 9. https://doi.org/10.3389/fnhum.2015.00137.

Grant, J. A., Courtemanche, J., Duerden, E. G., Duncan, G. H., \& Rainville, P. 2010. Cortical thickness and pain sensitivity in zen meditators. Emotion, 10(1): 43-53. https://doi.org/10.1037/a0018334.

Jadhav, N., Manthalkar, R., \& Joshi, Y. 2017. "Effect of meditation on emotional response: An EEG-based study." Biomedical Signal Processing and Control 34: 101-113. https://doi.org/10.1016/j.bspc.2017.01.008.

Lazar, S. W., Kerr, C. E., Wasserman, R. H., Gray, J. R., Greve, D. N., Treadway, M. T., ... Fischl, B. 2005. "Meditation experience is associated with increased cortical thickness." Neuroreport 16(17): 1893-1897.

Lee, Y.-H., Shiah, Y.-J., Chen, S. C.-J., Wang, S.-F., Young, M.-S., \& Lin, C.-L. 2015. "Improved Emotional Stability in Experienced Meditators with Concentrative Meditation Based on Electroencephalography and Heart Rate Variability." The Journal of Alternative and Complementary Medicine 21(1): 31-39. https://doi.org/10.1089/acm.2013.0465.

Lutz, A., Greischar, L. L., Rawlings, N. B., Ricard, M., \& Davidson, R. J. 2004. "Long-term meditators self-induce high-amplitude gamma synchrony during mental practice." Proceedings of the National Academy of Sciences, 101(46): 16369-16373. https://doi.org/10.1073/pnas.0407401101.

Lutz, A., Slagter, H. A., Rawlings, N. B., Francis, A. D., Greischar, L. L., \& Davidson, R. J. 2009. Mental Training Enhances Attentional Stability: Neural and Behavioral Evidence. Journal of Neuroscience 29(42): 1341813427. https://doi.org/10.1523/JNEUROSCI.1614-09.2009.

Tang, Y.-Y., Hölzel, B. K., \& Posner, M. I. 2015. “The neuroscience of mindfulness meditation.” Nature Reviews Neuroscience 16(4): 213-225. https://doi.org/10.1038/nrn3916. 